\title{
The scaling and dynamics of a projectile obliquely impacting a granular medium
}

\author{
Dengming Wang, Xiaoyan Ye, and Xiaojing Zheng ${ }^{\mathrm{a}}$ \\ Key Laboratory of Mechanics on Disaster and Environment in Western China, The Ministry of Education of China, and \\ Department of Mechanics and Engineering Science, School of Civil Engineering and Mechanics, Lanzhou University, Lanzhou, \\ Gansu 730000, China
}

Received 8 June 2011 and Received in final form 10 October 2011

Published online: 30 January 2012 - C EDP Sciences / Società Italiana di Fisica / Springer-Verlag 2012

\begin{abstract}
In this paper, the dynamics of a spherical projectile obliquely impacting into a two-dimensional granular bed is numerically investigated using the discrete element method. The influences of projectile's initial velocities and impacting angles are mainly considered. Numerical results show that the relationship between the final penetration depth and the initial impact velocity is very similar to that in the verticalimpact case. However, the dependence of the stopping time on the impact velocity of the projectile exhibits critical characteristics at different impact angles: the stopping time approximately increases linearly with the impact velocity for small impact angles but decreases in an exponential form for larger impact angles, which demonstrates the existence of two different regimes at low and high impact angles. When the impact angle is regarded as a parametric variable, a phenomenological force model at large impact angles is eventually proposed based on the simulation results, which can accurately describe the nature of the resistance force exerted on the projectile by the granular medium at different impact angels during the whole oblique-impact process. The degenerate model agrees well with the existing experimental results in the vertical-impact cases.
\end{abstract}

\section{Introduction}

The dynamical processes of a granular assembly under the impact of a projectile is widely present in Nature, such as the particle-bed collision in aeolian sand transport, the erosion of land surface, the formation of footprint on the beach, the landing of lunar vehicles, etc. Among these processes, the impact craters on the Moon and planets is one of the most typical phenomenon. However, as the formation of impact craters is too complex to be observed directly, astronomers and geologists usually infer such process and its basic mechanical behaviors through the morphology of the impact crater and the penetration depth of the projectile. So such investigations about the collision of a rigid projectile into a granular material provide a beneficial exploration to the penetration dynamics of a projectile and the scaling laws in the formation of impact craters on the Moon and planets.

Prior experimental investigations about the impact craters mainly focus on the hypervelocity projectile impacting into the target with a velocity on the order of a kilometer per second [1-3]. Recently, experiments based on low-energy impacts in granular materials, which may be readily studied in laboratory settings, have presented

a e-mail: xjzheng@lzu.edu.cn some meaningful phenomenon that is very similar to that observed in such hypervelocity impact experiments $[4,5]$. And it is suggested that the final stages of the excavation of the crater under these two impact conditions may have some analogies, particularly the crater morphology and corresponding dynamical behaviors $[5,8]$. Therefore, a large number of experiments [4-16], numerical simulations $[17,18]$ and theoretical models $[12-15,17]$ on lowvelocity impacts have been performed to explore the formation of the impact crater in granular media and attempt to propose some universal scaling describing the dimensions of the impact crater.

Recently, many experiments and simulations on lowvelocity impacts have focused on the penetration depth before stopping when a projectile impacts into a granular material. These investigations have mainly considered the scaling law of the penetration depth of the projectile with various system parameters, which currently becomes one of the key issues in understanding the dynamics of impacting a granular material. Despite the fact that recent progresses have been made on the penetration process of a rigid sphere into a granular material and various scaling laws about the penetration depth have been proposed under different system parameters, dimensions, impact conditions, etc. $[4,6,9,12-18]$, such impact dynamics is still difficult to be understood well as it involves both complex 
friction and collision processes. So far, the scaling law of the penetration depth with the impact velocity is still a controversial issue.

The stopping time of a projectile perpendicularly impacting into a granular medium displays a unique feature $[12,15,16,18]$ : for a low impact velocity, the stopping time decreases with the impact velocity, while for a velocity larger than a characteristic velocity (the characteristic velocity is defined as the velocity when the stopping time reaches an asymptotic plateau value), it is almost independent of the impact velocity. Although some existing experimental results have presented various characteristic times (the asymptotic plateau time) and characteristic velocities, the relationship between stopping time and impact velocity seems to exhibit a similar scaling law.

Based on the existing impact experimental results, some phenomenological models have been proposed to account for the dynamics of the projectile during the whole collision process. These models show that the resistance forces on the projectile mainly involve several terms: a hydrodynamic drag force proportional to the square of the velocity $[12,14,15,17,18]$ (which plays a dominant role for high-velocity impact and deep penetration), a static drag force depending on the depth $[14,15,17,18]$ (which plays a dominant role for low-velocity impact and shallow penetration) and a drag force which includes a term proportional to the velocity $[10,13]$. It is very difficult to extract these force terms from experiments directly. Recently, Goldman and Umbanhowar [12] measured the force with an accelerometer inside the impact sphere, and revealed that a force proportional to the square of velocity indeed exists during the first penetration stage at high speed and shallow depth. However, these studies mentioned above mainly focus on the vertical-impact cases.

Compared with the vertical-impact cases, researches on the dynamics of a projectile obliquely impacting into a granular medium and on the formation of impact craters are quite limited. Experiments and simulations [19-23] have been carried out to reveal the ejection process (splash function) for the oblique impact of a sphere into a granular bed from the viewpoint of aeolian sand transport, where the projectile commonly has a similar diameter as the grains in the target bed. However, for projectile's size much larger than that of target grains, the formation and morphology of the impact crater, as well as the dynamics of the projectile are not yet fully understood. Nishida et al. [24] carried out an oblique-impact experiment, and found that the projectiles have three different post-impact motions. Zheng et al. [11] examined the morphology of the impact craters formed by steel balls obliquely impacting a granular medium and proposed two scaling laws on the length and width of impact craters. Lee et al. [25] established a theoretical model of a rigid body obliquely impacting into a granular medium, based on the dynamic frictional force from vertical-impact results $[15,17]$ and the static resistance forces from quasi-static experiments $[26$, 27 . It was the first mathematical model proposed describing a rigid projectile dropping into granular matter at a specific angle. However, whether the resistance forces of the projectile in obliquely impacting a granular medium can be obtained by simply summing the dynamical friction force and the static resistance force, still need to be further confirmed.

To our knowledge, current investigations on dynamical behaviors of obliquely impacting into a granular bed is quite preliminary, especially with respect to the penetration depth, the stopping time, the resistance force on the projectile, etc. These important quantities are difficult to be measured directly using the existing measurement methods or experimental conditions. In this paper, we numerically investigate the dynamics of an oblique impact into a two-dimensional granular medium using the discrete element method, and the influences of initial velocity and impact angle on the penetration depth and stopping time of the projectile are mainly discussed. Finally, based on the simulation results, a phenomenological model is proposed to describe the resistance forces exerted on the projectile in oblique impact cases when the impact angle is larger than the critical value.

\section{Model and method}

We prepare a granular target composed of 15000 mixedsized particles, which includes 12600 small particles (diameter $d=0.456 \mathrm{~cm}$ and mass $m=0.049 \mathrm{~g}$ ) and 2400 large particles $(d=0.635 \mathrm{~cm}$ and $m=0.097 \mathrm{~g})$. The bidisperse mixture is employed to avoid crystallization. It is known that the way of generating the targets has influence to the final material [28], which is significant mainly for cohesive aggregation, but also for some non-cohesive cases. Here the particles are all randomly generated within a rectangular container with a width of $210 d_{g}$, where $d_{g}=0.485 \mathrm{~cm}$ is the mean particle diameter. The initial locations are set to prevent initial interactions. Then the gravitational forces are applied to all particles and tracing the motions of particles until the final stable state is achieved. For each impact event, the same bed height $\left(70 d_{g}\right)$ and area fraction $(\phi=0.804)$ are employed, the projectile with the diameter $d_{b}=4.46 \mathrm{~cm}$ and mass $m_{b}=32.2 \mathrm{~g}$ will impact the target bed with different velocities ranging from $0.5 \mathrm{~m} / \mathrm{s}$ to $4 \mathrm{~m} / \mathrm{s}$ and impact angles from $5^{\circ}$ to $90^{\circ}$, the initial angular velocity is ignored. It is worthwhile noting that the size of the container is large enough to avoid any boundary effects during the penetration [29].

The impact simulations are carried out using the discrete element method (DEM), which was originally developed by Cundall and Strack [30]. In this method, the position of each particle in the system is obtained by integrating twice with respect to time in Newton's second law of motion. DEM is in fact a time-driven soft-particle method that allows for two particles' interpenetrating so as to mimic particles' deformations. The governing equations of motion for particle $i$ are

$$
\begin{aligned}
m_{i} \frac{\mathrm{d} \vec{v}_{i}}{\mathrm{~d} t} & =m_{i} \vec{g}+\sum_{j}\left(\vec{F}_{n, i j}+\vec{F}_{s, i j}\right), \\
I_{i} \frac{\mathrm{d} \omega_{i}}{\mathrm{~d} t} & =\sum_{j}\left(T_{i j}+M_{i j}\right),
\end{aligned}
$$



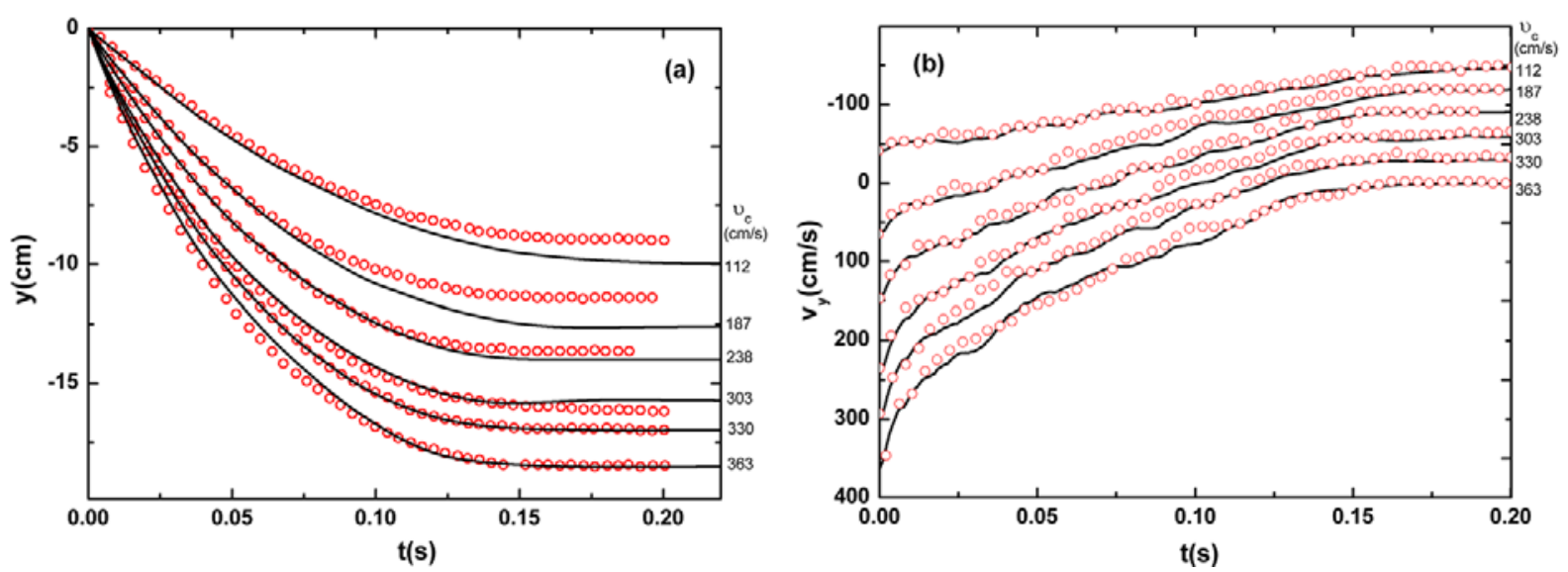

Fig. 1. (a) The position $y(t)$ and (b) the normal velocity $v_{y}(t)$ of the projectile versus time for different impact velocities, from experiment $(\circ)$ (conducted by [16]), solid lines are the results of simulations. The ordinate for the graph $v_{y}(t)$ for each successive impact velocity $v_{c}<363 \mathrm{~cm} / \mathrm{s}$ is shifted by $30 \mathrm{~cm} / \mathrm{s}$ for clarity.

where $m_{i}$ and $I_{i}$ are the particle mass and moment of inertia, $\vec{v}_{i}, \omega_{i}$ and $I_{i}$ are the velocity vector, angular velocity and the moment of inertia of particle $i$, respectively; $\vec{F}_{n, i j}$ and $\vec{F}_{s, i j}$ are the normal and tangential force of particle $j$ to particle $i, T_{i j}=\vec{R}_{i} \times \vec{F}_{s}$ is the tangential contact moment and $M_{i j}$ is the rotation frictional moment; $\vec{R}_{i}$ is the vector directed from the center of particle $i$ to the contact point.

The interactions between two particles can be described using the relative distances between the centers of the two particles. The contact force between particles $i$ and $j$, which can be decomposed into the normal contact force and the tangential contact force, is given by

$$
\begin{aligned}
& \vec{F}_{n}=-k_{n} \vec{\delta}_{n}-m_{r} \gamma_{n} \vec{v}_{n} \\
& \vec{F}_{s}=\frac{\vec{\delta}_{t}}{\left|\vec{\delta}_{t}\right|} \min \left[k_{t}\left|\vec{\delta}_{t}\right|-\gamma_{t} m_{r}\left|\vec{v}_{t}\right|, \mu\left|\vec{F}_{n}\right|\right] .
\end{aligned}
$$

Here, $\vec{\delta}_{n}$ is the overlap between two particles, $\vec{v}_{n}$ and $\vec{v}_{t}$ are the normal and tangential relative velocities, $\vec{\delta}_{t}$ is the tangential deformation vector, $m_{r}$ is the equivalent mass $\left(m_{r}^{-1}=m_{A}^{-1}+m_{B}^{-1}\right.$ is the equivalent mass of $\mathrm{A}$ and $\left.\mathrm{B}\right)$, $k_{n}=k_{t}=3200 \mathrm{~kg} \mathrm{~s}^{-2}$ are the stiffness coefficients which are proportional to Young' modulus, $\gamma_{n}=10^{4} \mathrm{~s}^{-1}$ and $\gamma_{t}=8 \times 10^{3} \mathrm{~s}^{-1}$ are viscoelastic constants and $\mu=0.28$ is the static friction coefficient. Ciamarra et al. [16] have obtained simulation results consistent with experimental results using the above-mentioned parameters. Therefore, in this work we also choose the same parameters as in ref. [16].

\section{Results and discussion}

\subsection{Model validity}

In order to evaluate the effectiveness of the numerical model, we have performed some simulations under conditions very similar to an existing experiment [16], which mainly focuses on the dynamical process of vertical impact in a $2 \mathrm{D}$ granular medium. It can be found that our simulation results about varying features of the penetration depth and the velocity with time are consistent with the experimental results for all the impact velocities, as shown in fig. 1. In addition, three distinct regimes of projectile's motion are also observed in our simulations: impact, penetration and collapse. Due to the inhomogeneous and anisotropic characteristics of the granular medium, the velocity of the projectile with time exhibits a clear fluctuating phenomenon, which may be induced by a stickslip feature of the resistance forces on the projectile [26].

However, slightly quantitative differences in the maximum penetration depth and the velocity of the projectile are observed between the simulation and experiment results. These differences might be induced by the randomness of the bed generation or the initial position of the projectile. Furthermore, the electric field forces caused by friction or collision between particles may have an influence on the trajectory and velocity of the projectile in the experiments $[31,32]$. The comparison between experiments and the numerical simulations under similar conditions indicates that the numerical model in this paper can effectively predict the dynamical process of the impact crater in the granular medium. Note that a slight difference among the dropping $x$-positions of the projectile leads to a weak difference in its acceleration even if the penetration depth and the stopping time are very similar. The results obtained in this paper are all the statistical averaged results over six different $x$-positions.

\subsection{Basic dynamic process of an oblique impact}

In order to understand the basic dynamical process of a projectile obliquely impacting into a granular material, we analyze the evolution of the velocity field and the trajectories of the projectile at different impact angles based on the discrete element method. Figure 2 shows the evolution of velocity field at the impact angle $\theta=45^{\circ}$ and impact 


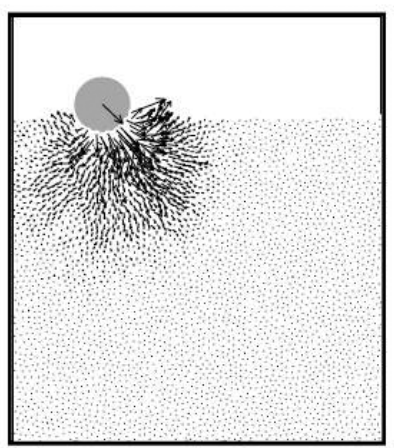

$0.00603 \mathrm{~s}$

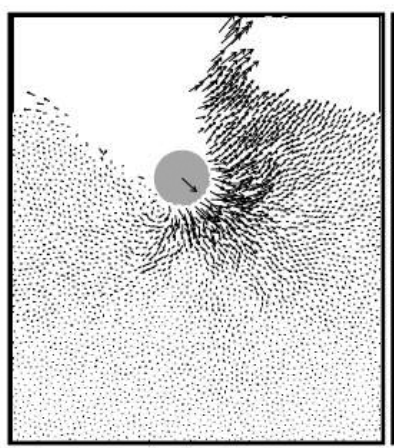

$0.04945 \mathrm{~s}$

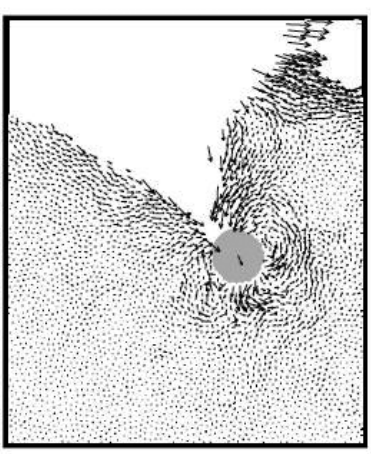

$0.14478 \mathrm{~s}$

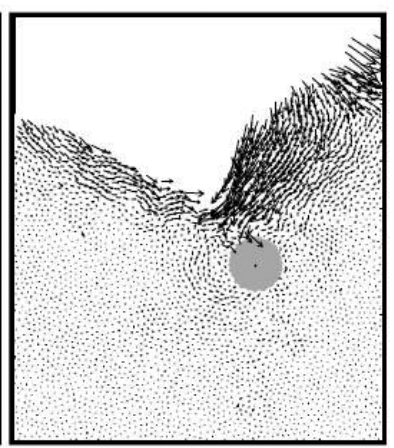

$0.19453 \mathrm{~s}$

Fig. 2. Snapshots of the velocity field in the formation of a crater when the impact velocity is $3 \mathrm{~m} / \mathrm{s}$ and the impact angle is $45^{\circ}$.

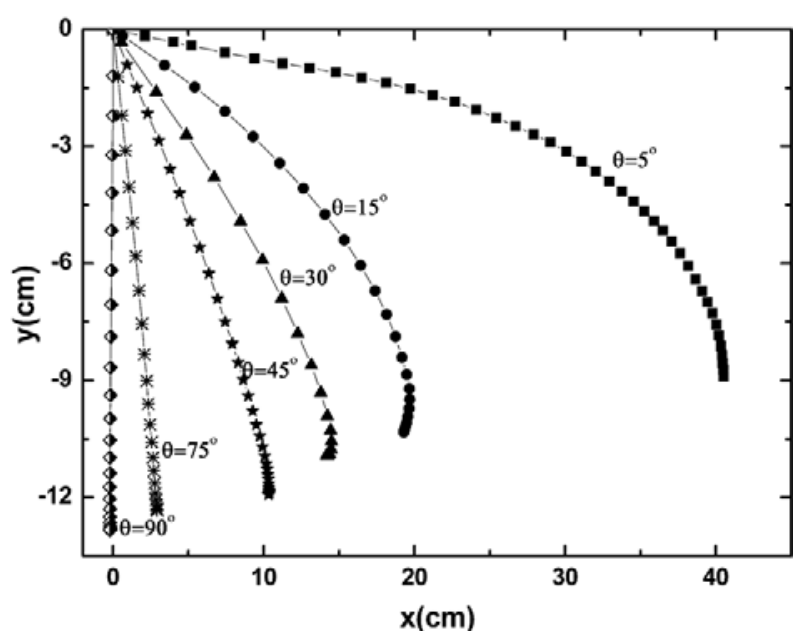

Fig. 3. Trajectories of the projectile at different impact angles $\left(v_{c}=2 \mathrm{~m} / \mathrm{s}\right)$.

velocity $v_{c}=3 \mathrm{~m} / \mathrm{s}$ in an oblique-impact case. Similar to the vertical-impact case [16], three distinct regimes of projectile's motion can also be observed: impacting, penetrating and collapsing. However, as the projectile may commonly move a distance along the horizontal direction after impacting, the collapses of bed grains mainly occur in the forward side of the impact point, which leads to the formation of an asymmetrical impact crater as observed in the experiment [11].

Figure 3 shows the trajectories of the projectile at different impact angles during the whole impact process. If the impact velocity is fixed at $v_{c}=2 \mathrm{~m} / \mathrm{s}$, it can be found that the projectile's trajectory exhibits a critical transition from linear to nonlinear gradually with the decreasing of the impact angle of the projectile. At the same time, we also find that the impact velocity exhibits a similar influencing feature on the moving trajectory of the projectile at a given impact angle. Such critical phenomenon shows that the impact angle and the impact velocity play very important roles in the dynamics process of a projectile obliquely impacting into a granular bed. In addition, the vertical components of the impact velocities correspond- ing to the critical transition of trajectories are all around $1.12 \mathrm{~m} / \mathrm{s}$ at different angles, which indicates that it may be the principal factor affecting the dynamics of the projectile. Note that there exists a circuity at the end of the oblique penetration, this may be induced by the fact that the transient impact crater collapses and then the ejected particles deposit on the projectile again.

Due to the combined influences of many factors, such as impact velocity, impact angle, system parameters, etc, the oblique-impact problems in a granular medium exhibit more complex dynamical behaviors comparing to those in vertical-impact cases.

\subsection{Penetration depth}

When a projectile vertically impacts into a granular material, the dependence of the penetration depth on the impact velocity and corresponding scaling laws have intensely been considered in previous investigations $[9,12-$ 17]. Here we mainly focus on the penetration depth of the projectile and the involved scaling law if a sphere obliquely impacts into a granular material. The movement of the sphere inside a granular material can be decomposed into a horizontal and a vertical component. Then the penetration depth is commonly defined as the distance measured from the original impact point to the bottom of the impacting sphere. This penetration depth also has a horizontal and a vertical component, called $d_{x}$ and $d_{y}$, respectively. Indeed, most of the ejected grains may redeposit into the granular bed and apply a pressure on the projectile during the collision process, which lead to a downward movement when the projectile is closer to stopping. This small rebound effect may occur in many impacting cases but it is ignored in the statistics of the final penetration depth.

The horizontal and vertical penetration depths of the sphere, which are averaged over 6 different impact positions on the target bed, all approximately linearly increase with the impact velocity at all the impact angles, as shown in fig. 4(a) and (b). Fittings to the two sets of data are shown as dashed lines and all the sets of the data with 

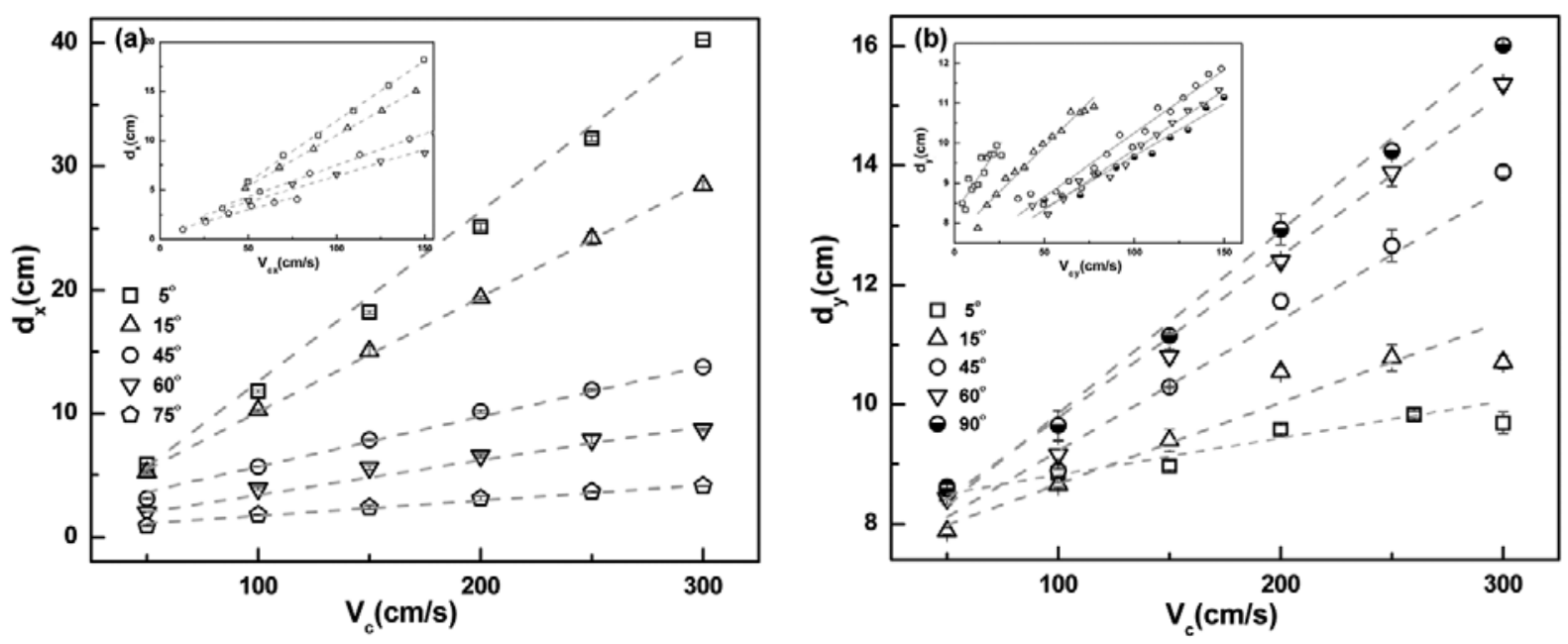

Fig. 4. (a) Horizontal penetration length $d_{x}$ and (b) vertical penetration depth $d_{y}$ versus initial impact velocities at specific impact angles: $\theta=5^{\circ}, \theta=15^{\circ}, \theta=45^{\circ}, \theta=60^{\circ}, \theta=75^{\circ}$ and $\theta=90^{\circ}$; the dashed lines are the fitting lines. Inset: (a) horizontal penetration length $d_{x}$ and (b) vertical penetration depth $d_{y}$ versus the horizontal velocity component $v_{c x}$ and the vertical velocity component $v_{c y}$, respectively, at different impact angles; the dashed lines are the fitting lines.

nonzero intercepts follow a linear relationship. It is also found that the error of $d_{x}$ and $d_{y}$ under different impact positions are smaller than $6 \%$, which shows that the impact position of the projectile has little influence on such linear relationship.

Summarizing the simulating results of fig. 4, we write the variation of the penetration depths, $d_{x}, d_{y}$ with the impact velocity $v_{c}$ as the following scaling laws:

$$
\begin{aligned}
& d_{x}=\lambda_{x 1} v_{c}+\lambda_{x 2}, \\
& d_{y}=\lambda_{y 1} v_{c}+\lambda_{y 2},
\end{aligned}
$$

where $\lambda_{x 1}, \lambda_{y 1}$ are slopes, having dimensions of time; $\lambda_{x 2}$, $\lambda_{y 2}$ are intercepts, having dimensions of length. These fitting parameters are all functions of the impact angle $\theta$, which also may be influenced by the system parameters, such as mixture ratio, ratio of particle diameter, etc. If the variation of packing fraction is small, it can be confirmed that the scaling of penetration depth we proposed here is almost independent of the choice of system parameters although such fitting parameters may be quite different. Under the conditions given in this paper, the values of these four parameters at different impact angles are listed in table 1 of appendix $\mathrm{A}$.

Based on eqs. (5), (6) and table 1 in appendix A, the slopes and intercepts in the above scaling laws are not constants within the whole range of impact angles. The slope $\lambda_{x 1}$ in length scaling decreases with $\theta$ but the slope $\lambda_{y 1}$ increases with $\theta, \lambda_{x 1} / \cos (\theta)$ and $\lambda_{y 1} / \sin (\theta)$ are both decreasing functions of $\theta$, which means $d_{x}$ and $d_{y}$ decrease with increasing $\theta$ for fixed velocity components $v_{c x}$ and $v_{c y}$, as shown in the inset of figs. $4(\mathrm{a})$ and (b). This demonstrates that the total displacement of the projectile is a decreasing function of the impact angle $\theta$. It is speculated that the force chains in a granular medium may be strengthened with the increasing of the depth and then more energy is needed to destroy such spatial struc- tures. The projectile at small impact angles may commonly move inside the upper layer of the granular bed, so the required energy to destroy the force chains inside a granular medium is relatively small, which leads to a larger displacement of the projectile. Therefore, the influence of the impact angle on the penetration depth is more significant at small impact angels. However, it can be found that the impact angle has little effect on the vertical penetration at large impact angles, as shown in fig. 4(b).

It can be seen that the scaling of penetration depth under oblique impacts shows characteristics linearly changing with impact velocity. In fact, a similar scaling, the penetration depth with impact velocity scaled as $d \sim v_{c}$, has also been proposed in some vertical-deep-impact experiments $[12,13]$ where the maximum penetration depths were much greater than one sphere diameter, and also in a quasi-2D experiment where disks were confined between narrow side walls [16]. When the scaling we proposed degrades into a vertical-impact case, $d_{x}$ tends to zero and $d_{y}$ increases linearly with impact velocity $v_{c}$, which is qualitatively consistent with these experimental results. The simulating trajectories of the projectile in vertical-impact cases (see fig. 1) also reveal that the penetration depth from the above relationship is in quantitative agreement with similar experiments [16].

\subsection{Stopping time}

The stopping time of a sphere vertically impacting into a granular material exhibits a striking behavior: it is a decreasing function of the impact velocity with an asymptotic plateau at large enough impact velocities $[12,15,16$, 18] For the oblique-impact case, in order to investigate the relationship between the stopping time and the impact velocity, we define the time interval, from the projectile contact with the target to the instantaneous point when 


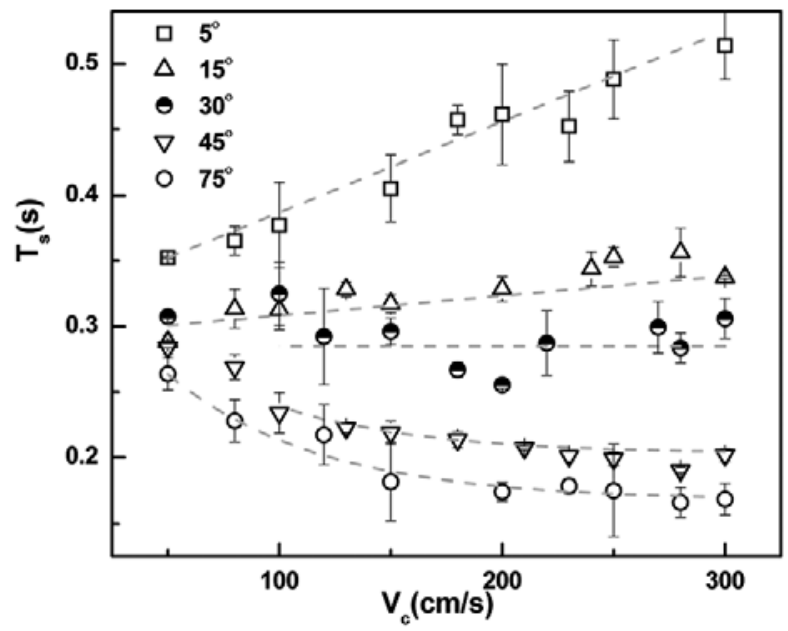

Fig. 5. The stopping time versus the impact velocity at different impact angles, the dashed lines are the fitting lines.

the moving horizontal velocity becomes zero, as the horizontal stopping time. And the vertical stopping time is also defined using a similar approach. Here we neglect the circuity effect of the projectile at the end of the penetration process. The larger one of these two stopping times, $T_{s}$, is defined as the stopping time of the projectile during the entire impact process.

The scaling curves of the stopping time $T_{s}$ versus the impact velocity $v_{c}$ at different impact angles are shown in fig. 5. The figure shows that the varying trend of $T_{s}$ with $v_{c}$ exhibits two different regimes at low and high impact angles, and that there is a critical impact angle, which approximately equals $30^{\circ}$ under these conditions. When the impact angle is larger than this critical value, the stopping time decreases with $v_{c}$ at low impact velocities and shows an asymptotic plateau at large enough impact velocities, which is a feature similar to that in the vertical-impact cases $[12,15,18]$. However, if the impact angle is smaller than this critical angle, the stopping time becomes an approximately linearly increasing function of the impact velocity. This qualitative inconsistence in the scaling of the stopping time may be essentially determined by the existence of two different regimes of the resistance force on the projectile at low and high impact angles, which will be discussed in detail in the following section. The critical angle $\left(30^{\circ}\right)$ and the vertical component of characteristic velocity (around $1.12 \mathrm{~m} / \mathrm{s}$ ) may be related to the system parameters of the granular medium, such as density ratio, diameter ratio, packing fraction, etc. [24].

Based on the above analysis, it can be found that the stopping time decreases with increasing velocity and follows a negative exponential trend when the impact angle $\theta$ is larger than the critical angle $30^{\circ}$, otherwise it increases linearly with $v_{c}$. According to this phenomenon, we propose a scaling of the stopping time with the impact velocity $v_{c}$ as follows:

$$
T_{s}= \begin{cases}\eta_{1} e^{-\eta_{2} v_{c}}+\eta_{3}, & \theta>30^{\circ}, \\ \chi_{1} v_{c}+\chi_{2}, & \theta<30^{\circ},\end{cases}
$$

where $\eta_{1}, \eta_{2}, \eta_{3}, \chi_{1}$ and $\chi_{2}$ are some parameters related to the impact angle $\theta$. Table 2 in appendix A provides some fitting values of these parameters at the different impact angles based on our simulation results. Performing a series of numerical simulations, we also found that these fitting parameters may be influenced by the many factors of the granular material, such as mixture ratio, diameter ratio, packing fraction, etc. However, if the change of the diameter ratio and diameter ratio is small, the corresponding variation of the initial packing fraction is also small, where the granular bed is still on a relative loose deposited status under gravity and the rebounding of the projectile does not occur significantly during the impact process. Under such conditions, the proposed scaling law here is also suitable even if the target bed changes.

When the impact angle is larger than its critical value, we can find that the stopping time is a decreasing function of the impact velocity $v_{c}$ with an asymptotic plateau at large enough impact velocities. At small impact velocities, the projectile may penetrate more deeply with increasing the impact velocity relatively, where the gravity is commonly larger than the resistance force exerted on the projectile by the granular medium (this can be confirmed by the simulation results in sect. 3.5 ), so the initial energy may dissipate more quickly and then the stopping time becomes shorter. If the impact velocity is large enough, the surface of the sphere in contact with the granular media is expected to be essentially constant for a large fraction of the collision interval after the initial impact $[12,17]$, and then the stopping time is almost independent of $v_{c}$. The stopping time reaching an asymptotic plateau is related to the fitting parameters $\eta_{1}$ and $\eta_{2}$. As seen in table 2 in appendix A, $\eta_{1}$ decreases with $\theta$, whereas $\eta_{2}$ increases with $\theta$, this indicates that the stopping time reaches the plateau more easily at larger impact angles. The variation of the fitting parameter $\eta_{3}$ with $\theta$ further demonstrates that the characteristic time is a decreasing function of $\theta$. However, when the impact angle is smaller than the critical value, the projectile commonly moves inside the upper layer of the medium and the initial energy may dissipate more slowly, which leads to a linear increase of the stopping time with $v_{c}$. In addition, the simulation results reveal that the stopping time is a decreasing function of the impact angle at a specific impact velocity.

It is worth noticing that the stopping time in obliqueimpact cases is mainly influenced by the vertical component $v_{y}$ of the impact velocity at all impact angles in our simulation results. The existing theoretical investigation concludes that the stopping time is influenced by the horizontal velocity at small impact angles $\theta=15^{\circ}, 5^{\circ}[25]$. This difference can be attributed to the fact that this theoretical model neglects the angular velocity of the projectile, which has a significant variation in the oblique-impact cases, especially at small impact angles [24]. In addition, the difference of the force laws used in the calculation of the stopping time at small impact angles may be another important factor, which will be discussed in the following section. 


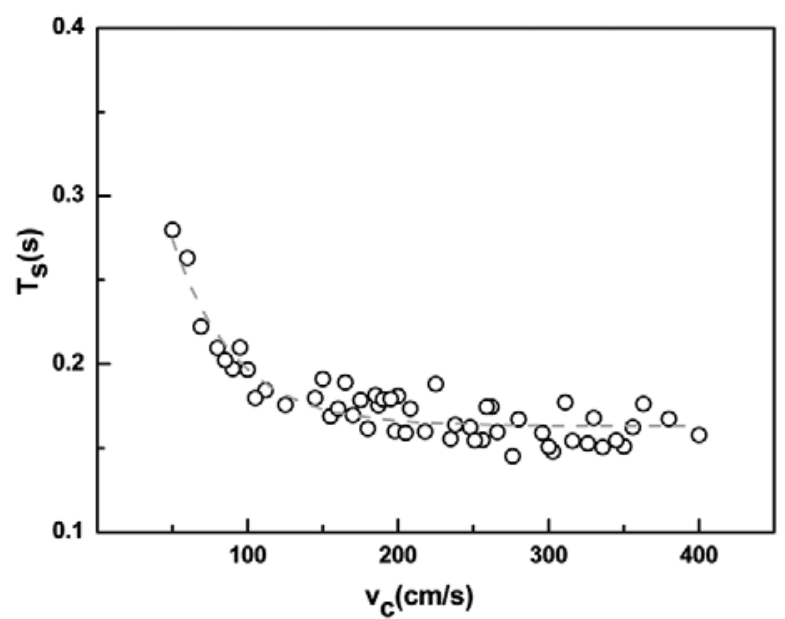

Fig. 6. The stopping time versus the impact velocity for a vertical impact, the dashed line is the fitting line.

Here the influence of the impact velocity on the stopping time of a projectile vertically impacting into a granular material is also performed, and we find that it can be well described using the reduced scaling of eq. (7) at $\theta=$ $90^{\circ}$, as shown in fig. 6 . The vertical-impact experiments show that the characteristic time is $\tau=(2 R / g)^{1 / 2}[15$, 16] or $\tau=\left(\rho_{b} / \rho_{g}\right)^{1 / 4}(2 R / g)^{1 / 2}$ [12] at an impact velocity larger than the typical characteristic velocity $V=$ $(2 g R)^{1 / 2}[12,15]$, and the $2 \mathrm{D}$ vertical simulation [18] reveals that the characteristic time is $\tau=\left(2 \rho_{b} R / \rho_{g} g\right)^{1 / 2}$ at an impact velocity larger than the characteristic velocity $V=\left(2 \rho_{b} g R / \rho_{g}\right)^{1 / 2}$. Our simulation result is qualitatively consistent with the experimental results $[12,15]$. The characteristic velocity is approximately $1 \mathrm{~m} / \mathrm{s}$ in this paper, which is slightly larger than $V=(2 g R)^{1 / 2}[13,15]$. The characteristic time is about $0.16 \mathrm{~s}$, which agrees well with the simulation results [16].

\subsection{Force law}

It is obvious that the variation of the penetration depth and the stopping time of the projectile with the impact angle discussed above is essentially completely related to the resistance force exerted on the projectile by the granular bed during the whole impact process. Based on our simulation results, we aim to propose a scaling law describing such resistance force with some phenomenological models, which can capture the average dynamics involved in the oblique-impact events.

As the destruction and reconstruction of the force chains inside a granular material are very common, the resistance force exerted on the projectile by a granular bed commonly exhibits a distinct fluctuating feature [16]. In oblique-impact cases, we find that such force fluctuations may exhibits different varying features with time at different impact angles. Figure 7 shows the time series of the horizontal and vertical components of the force on the projectile, $F_{x}$ and $F_{y}$, at two typical impact angles $\theta=5^{\circ}$, $45^{\circ}$. It is clear that at the larger impact angle (such as $\theta=45^{\circ}$ ), the force excreted on the projectile, especially its vertical component, may reach its peak value very rapidly and then decrease gradually until it stops, very similarly to what observed in a vertical-impact simulation [16]. Under this condition, the projectile commonly penetrates more deeply, so the initial energy inevitably dissipates faster as more intense restrictions from surrounding grains, which may lead to a relatively short stopping time. If the impact angle is small (such as $\theta=5^{\circ}$ ), the projectile commonly moves inside the top layer of the granular bed for a fixed impact velocity, as shown in fig. 3. This may lead to a relatively small deceleration of the projectile during the whole impact process, and thus it needs a longer collision duration as the particles in the bed make small rearrangements in response to the collapse of the transient crater.

The experimental and simulation studies in verticalimpact cases show that the force exerted on the projectile mainly depends on the position $y$ and the velocity $v$ of the projectile inside the granular material $[12,15,17,18]$. Based on a similar consideration, we mainly focus on the variation of the resistance force on the projectile at different impact angles, and the most important question is whether this resistance force can be expressed using an independent function of the projectile's position and velocity, respectively.

Firstly, at each impact angle, we examine the relationship between the horizontal resistance force and the corresponding horizontal velocity through nine different fixed depths $y=\{15,25,35,45,55,65,75,85,95\} \pm 1 \mathrm{~mm}$. For the sake of clarity, here we only provide the simulation results at three different impact angles, as shown in fig. 8(a) From the results, we can find that the horizontal resistance force is approximately quadratic in the horizontal velocity, i.e. $F_{v x}=\alpha_{x} v_{x}^{2}$, and it is more crucial that at each impact angle the proportionality factor $\alpha_{x}$ is almost constant for all nine different depths. This demonstrates that the projectile experiences a horizontal resistance force that is independent of the depth, which is similar to the inertial drag force proposed previously in vertical-impact problems $[15,17,33,34]$. Furthermore, in order to further examine the relationship between the horizontal resistance force and corresponding position of the projectile, we present the force term at a vanishing velocity as a function of depth, as shown in fig. 8(b). It can be found that the depth-related resistance force is linearly increasing with depth and this relationship is independent of velocity, which indicates a depthdependence of the Coulomb friction in the granular material $[15,35]$.

The above analysis shows that the horizontal force on the projectile can be conveniently expressed as an inertial term quadratic to the horizontal velocity plus a Coulomb friction term proportional to depth of the projectile. Using a similar method, we also analyze the varying feature of the vertical resistance force with the position and vertical velocity, as shown in fig. 9(a) and (b), and the difference is that gravity must be considered in this direction. From the results we find the vertical force on the projectile can 

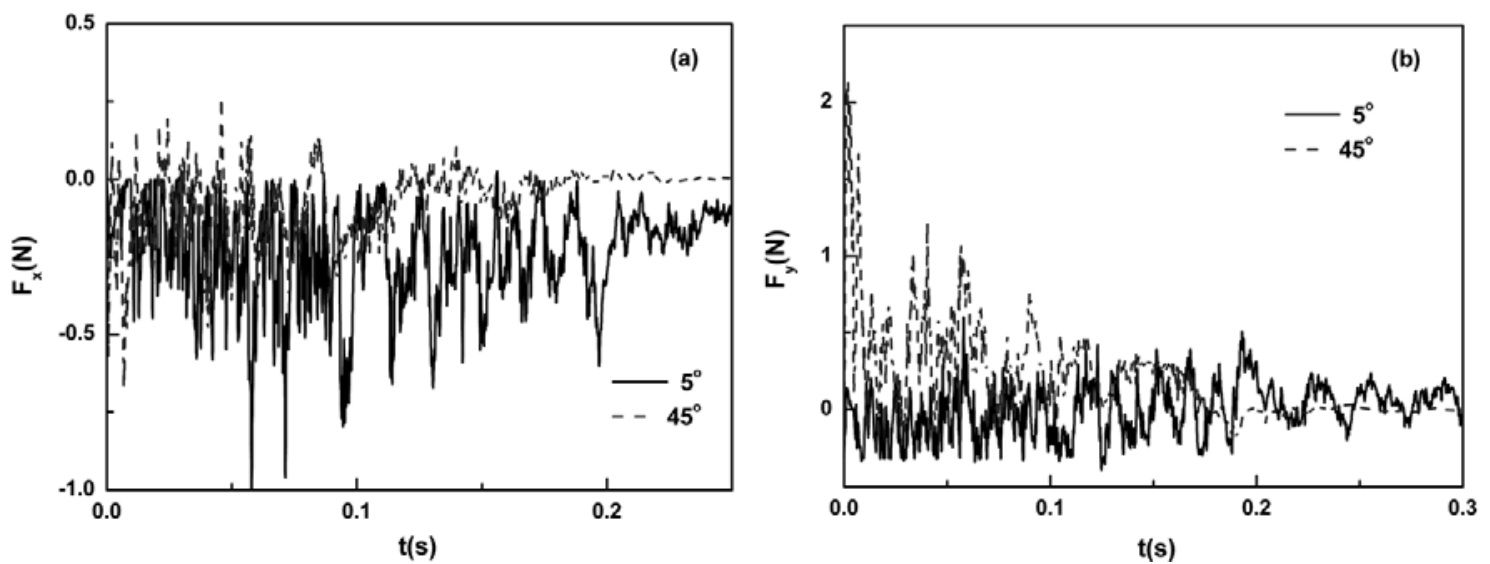

Fig. 7. The time series of (a) the horizontal force $F_{x}$ and (b) the vertical force $F_{y}$ on the projectile when the impact velocity is $2 \mathrm{~m} / \mathrm{s}$ and the impact angles are $5^{\circ}$ and $45^{\circ}$.
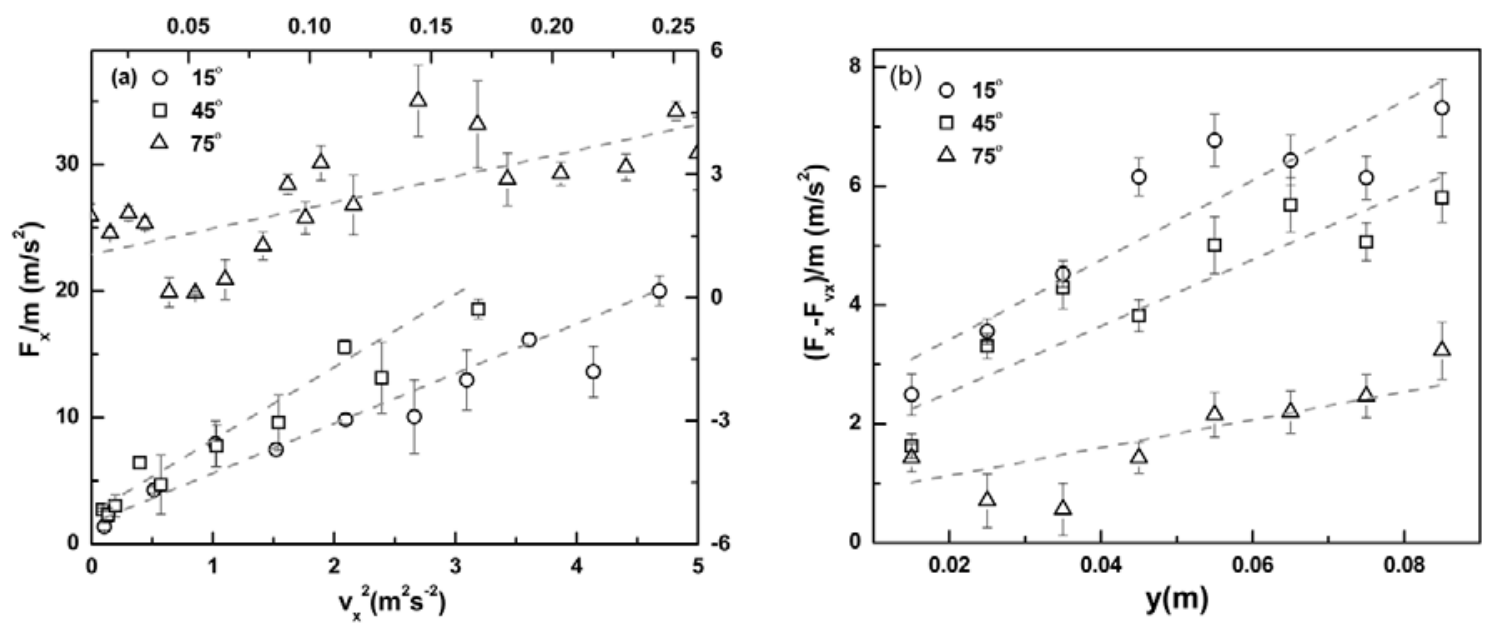

Fig. 8. (a) The square of the horizontal velocity component $v_{x}$ at a specific depth $y=0.025 \mathrm{~m}$ versus $F_{x} / m$ and (b) depth $y$ versus $\left(F_{x}-F_{v x}\right) / m$. In (a) there are two groups of Cartesian coordinates, the bottom and left coordinate axes are used to measure $v_{x}^{2}$ and $F_{x} / m$ at $15^{\circ}$ and $45^{\circ}$, the top and right ones are for $75^{\circ}$. The dashed lines are the fitting lines.
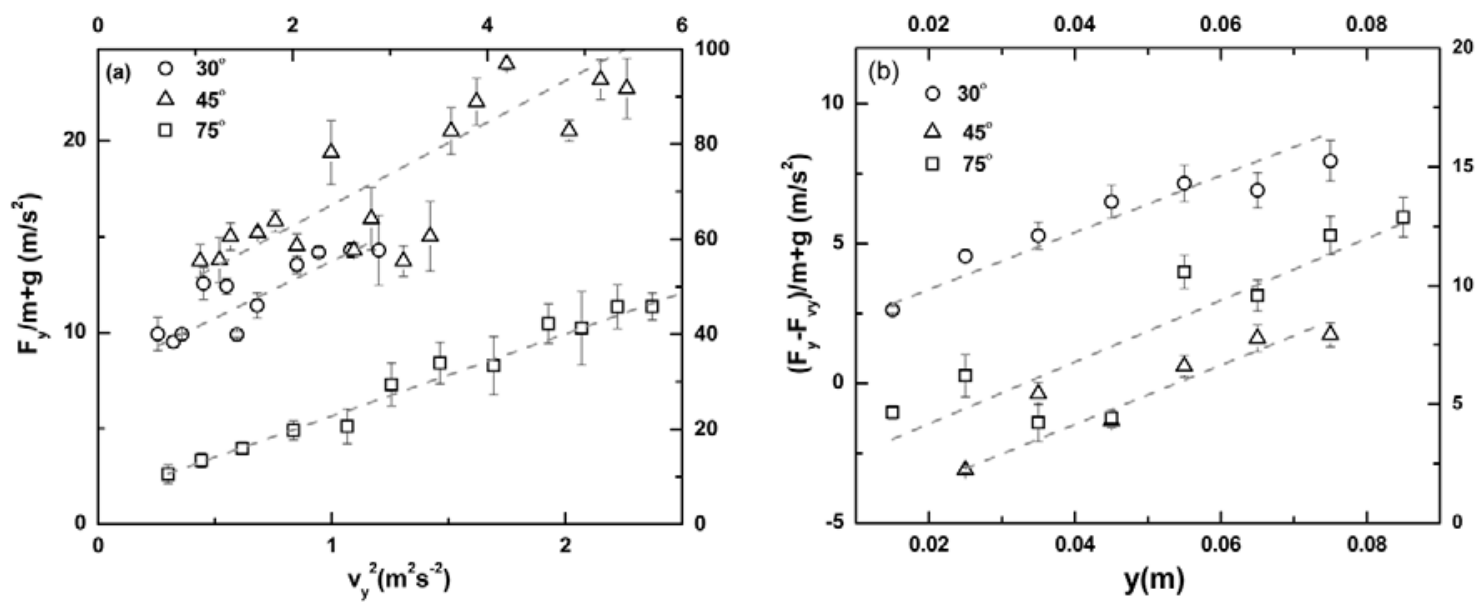

Fig. 9. (a) The square of the vertical velocity component $v_{y}$ at a specific depth $y=0.025 \mathrm{~m}$ versus the reduced acceleration $F_{y} / m+g$ of the impacting sphere and (b) the depth $y$ versus $\left(F_{y}-F_{v y}\right) / m+g$. In (a) there are two groups of Cartesian coordinates, the bottom and left coordinate axes are used to measure $v_{y}^{2}$ and $F_{y} / m+g$ at $30^{\circ}$ and $45^{\circ}$, the top and right ones are for $60^{\circ}$. In (b) the bottom and left coordinate axes are used to measure $y$ and $\left(F_{y}-F_{v y}\right) / m+g$ at $30^{\circ}$, the top and right ones are for $45^{\circ}$ and $75^{\circ}$. The dashed lines are the fitting lines. 


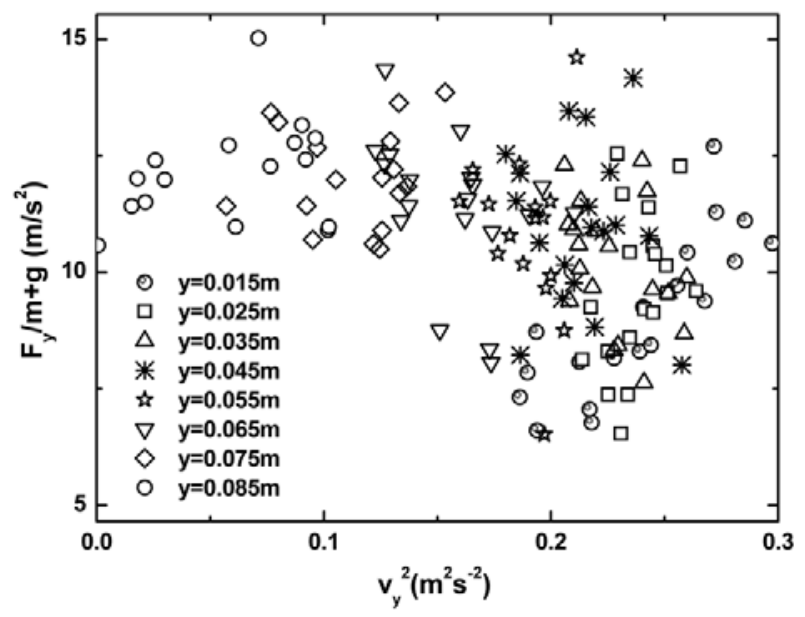

Fig. 10. The square of the vertical velocity component $v_{y}$ versus the reduced acceleration $F_{y} / m+g$ for different depths, at a specific angle $\theta=15^{\circ}$.

also be expressed as an inertial term quadratic to the vertical velocity plus a Coulomb friction term proportional to depth, and coupled to a gravity term. However, different from the horizontal force, such force law along the vertical direction is only suitable for large impact angles (here about $\theta \geq 30^{\circ}$ ). At small impact angles (such as $\theta=15^{\circ}$ ), this relationship is not satisfied any longer, as shown in fig. 10, which may lead us to conclude that the resistance force exerted on the projectile becomes very complex as discussed above.

The impact angle of the projectile is essentially an initial condition in an oblique-impact problem. However we find that the resistance force has much more complex dependence on the impact velocity if it is considered as an initial condition during the foundation of the force model, which may depend on the local orientation of the velocity direction and difficult be described by some simple fitting expressions. So in this paper, the impact angle of the projectile is only regarded as a parametric variable. Based on our simulation results, here we propose a phenomenological model describing the force exerted on the projectiles at large impact angles $\left(\theta \geq 30^{\circ}\right)$ in an oblique-impact case, expressed as follows:

$$
\begin{aligned}
& F_{x}=-\alpha_{x} v_{x}^{2}-f_{x}|y|, \\
& F_{y}=-m g+\alpha_{y} v_{y}^{2}+f_{y}|y|,
\end{aligned}
$$

where $\alpha_{x}, \alpha_{y}, f_{x}, f_{y}$ are some fitting parameters depending on the impact angle. The values of these parameters at different impact angles can refer to the statistical results of our simulations presented in table 3 of appendix A. It is easy to find that $\alpha_{y}$ and $f_{y}$ are all increasing with $\theta$, which implies that the vertical resistance force increases with $\theta$. Therefore, the stopping time presents a negative correlation with $\theta$ at a given impact velocity $v_{c}$ and the characteristic time is a decreasing function of $\theta$.

The force law proposed in this paper has a very similar form as given in the existing theoretical model of ref. [25] except two significant differences: one is that the coefficients in the force law are all constants in the theoretical model, but they are related to the impact angle in our simulations; the other is that the resistance force on the projectile can be expressed by two independent functions of position and velocity at all impact angles in the theoretical model, but it can be concluded in our simulations that such expression may be just suitable for larger impact angles. It can be seen that in this theoretical investigation, the force model is described by a composition of a dynamic experimental model along the vertical direction with the impact velocity of the order of $\mathrm{m} / \mathrm{s}$ and a quasi-static experimental result along the horizontal direction with the movement velocity of the order of $\mathrm{mm} / \mathrm{s}$. So it is required to further confirm whether the dynamics of a projectile obliquely impacting into granular media can be well described using such complex theoretical model. From our simulations it can be found that the resistance force on the projectile exhibits a very complex feature at small impact angles, where the projectile may be at a transient region showing a combination of rebound, penetration and motion parallel to the horizontal plane of the granular media, as observed in experiment [24].

In fact, as long as the variation of the initial packing fraction caused by the change of the target bed, such as mixture ratio, ratio of particle diameters, etc., is small, we have confirmed that the proposed scaling laws in this paper are still suitable although the fitting parameters will certainly be very different. However, if the variation of system parameters induces a significant increase of the packing fraction, the proposed scaling law may be no longer suitable due to the obvious rebounding phenomenon of the projectile during some impact processes, especially at small impact angles. Therefore, similar to what observed in a vertical-impact experiment [36], a critical packing state may also exist in the oblique-impact case, which may be a major factor influencing the changing feature of the resistance force on the projectile at different impact angles.

In order to check the validity of the proposed force law, we integrate the motion equations and compare the computed trajectories with those predicted by the discrete element method, as shown in fig. 11. It can be found that the computed results are well consistent with the simulation results. Furthermore, we perform a series of numerical simulations in a vertical-impact case, and find that the simulation results of the resistance force on the projectile are sufficient to allow the individual depth and velocitydependent terms to be isolated: an inertial term quadratic to the velocity and a Coulomb friction term proportional to the depth, as shown in figs. 12 and 13. It can be found that the force on the projectile can also be well described using a degenerated force law when the impact angle is set to $90^{\circ}$ in eqs. (8) and (9), which is well consistent with the existing theoretical models in vertical-impact cases [15, 18,37]. Through the analysis of simulation results we find that the fitting parameters in this degenerated model have the same order of magnitude as that proposed by Katsuragi [15]. Thus we achieve a unified description of the 

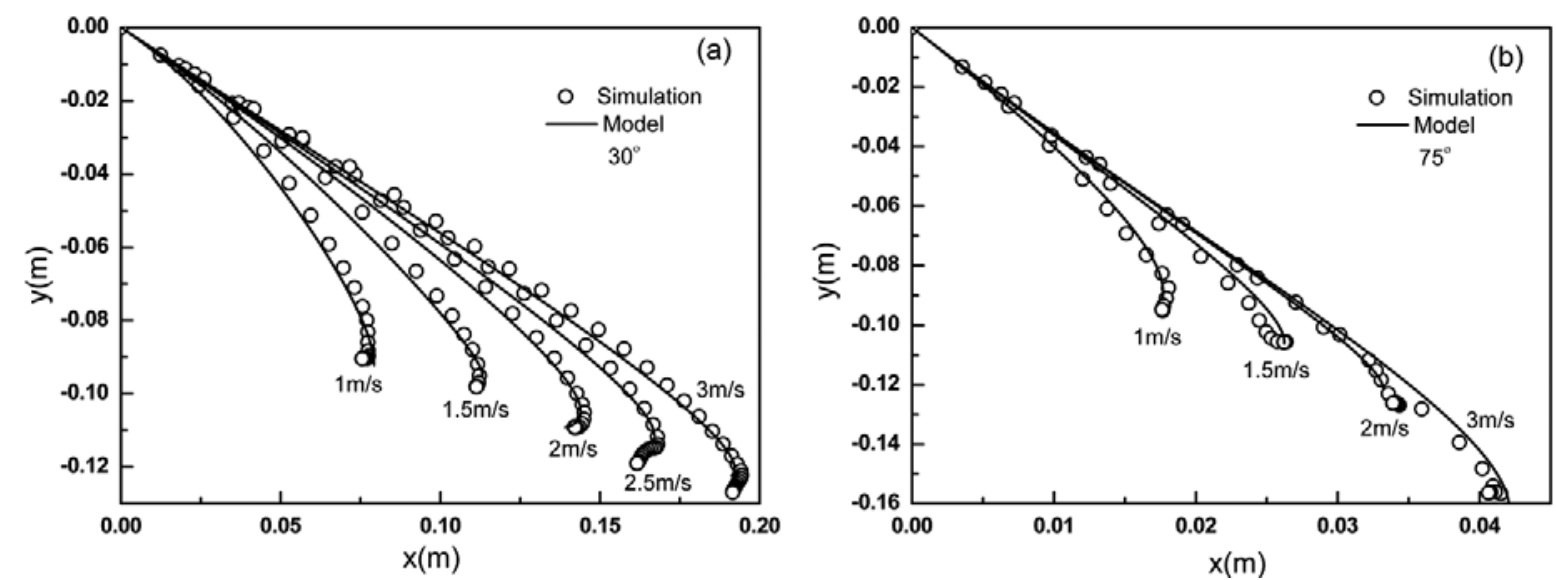

Fig. 11. Trajectories of the projectile for the impact angle (a) $30^{\circ}$, (b) $75^{\circ}$, from both simulation (o) and the model (solid lines).

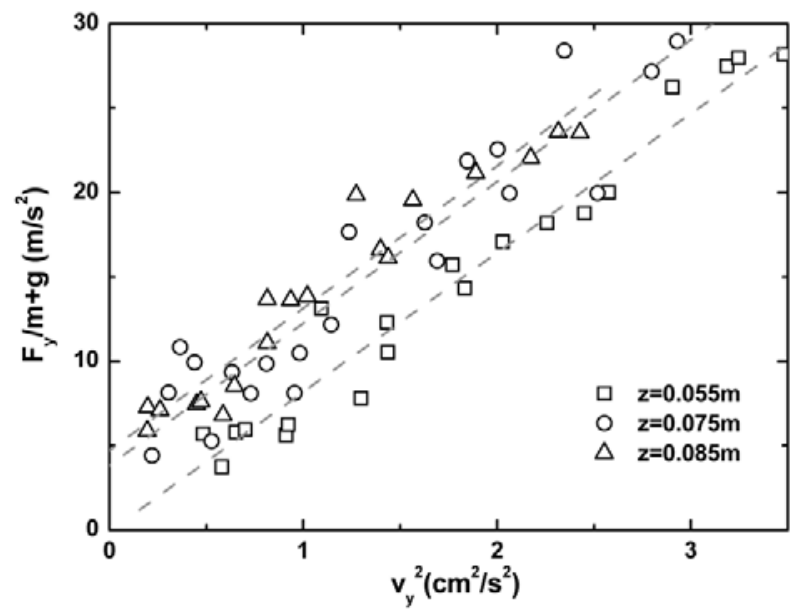

Fig. 12. Reduced acceleration $F_{y} / m+g$ of the impacting sphere versus its velocity squared, at a specific depth: $y=$ $0.055 \mathrm{~m}, y=0.075 \mathrm{~m}, y=0.085 \mathrm{~m}$.

resistance force on a projectile obliquely impacting into a granular material, which is also suitable for the verticalimpact case.

\section{Conclusions}

The dynamic process of a two-dimensional granular assembly subjected to the oblique impact of a spherical projectile is numerically investigated using the discrete element method, and the scaling of penetration depth and stopping time with the impact velocity and impact angle are also considered. The simulation results show that the dynamical behaviors of the oblique impact are more complex in comparison with the vertical impact. The horizontal and the vertical penetration depths of the projectile all show a linear relationship with the impact velocity at different impact angles, which is very similar to the scaling of the vertical impact. The relationships between the stopping time and the impact velocity demonstrate the existence of two different regimes at low and high im-

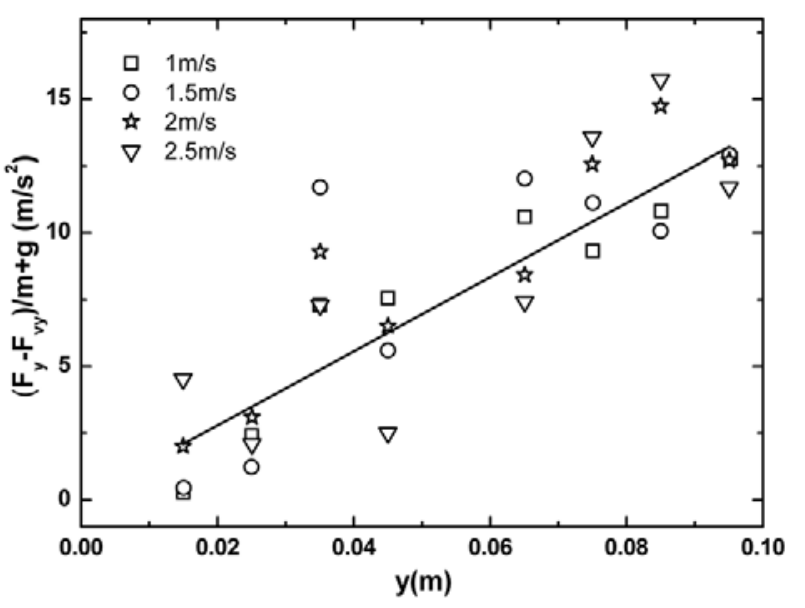

Fig. 13. $\left(F_{y}-F_{v y}\right) / m+g$ versus the depth $y$ of the projectile.

pact angles. When the impact angle is larger than a certain critical value (about $30^{\circ}$ ), the stopping time decreases with the increasing velocity and approximately tends to a constant at an impact velocity exceeding a characteristic value, which is very similar to that of the vertical impact. However, the stopping time increases linearly with the impact velocity when the impact angle is less than the critical value. Finally, when the impact angle is regarded as a parametric variable, we propose a phenomenological force model at large impact angles based on the simulation results, which can accurately describe the nature of the resistance force exerted on the projectile by the granular medium at different impact angles during the whole impact process. The degenerated model agrees well with the existing experimental results in the vertical-impact cases.

This work was supported by The State Key Development Program for Basic Research of China (No. 2009CB421304), National Natural Sciences Foundations of China (Grant Nos. 11022064, 10872082, 11072097), and Open Fund of State Key Laboratory of Structural Analysis for Industrial Equipment (No. GZ1009). The authors gratefully acknowledge these supports. 


\section{Appendix A.}

Table 1. Values of the slope and intercept of the horizontal and vertical penetration depths of eqs. (5) and (6) at different impact angles.

\begin{tabular}{ccccc}
\hline Impact angle (degrees) & $\lambda_{x 1}$ & $\lambda_{x 2}$ & $\lambda_{y 1}$ & $\lambda_{y 2}$ \\
\hline 5 & 0.13553 & -0.01677 & 0.0064 & 0.08188 \\
15 & 0.09257 & 0.00876 & 0.01193 & 0.07595 \\
30 & 0.05877 & 0.01848 & 0.01802 & 0.07257 \\
45 & 0.04042 & 0.01734 & 0.02309 & 0.06528 \\
60 & 0.02581 & 0.01289 & 0.02892 & 0.03112 \\
75 & 0.01339 & 0.00486 & 0.06472 \\
\hline
\end{tabular}

Table 2. Values of $\eta_{1}, \eta_{2}, \eta_{3}, \chi_{1}$ and $\chi_{2}$ of eq. (7) at different impact angles.

\begin{tabular}{cccccc}
\hline Impact angle (degrees) & $\eta_{1}$ & $\eta_{2}$ & $\eta_{3}$ & $\chi_{1}$ & $\chi_{2}$ \\
\hline 5 & - & - & - & 0.06193 & 0.31617 \\
15 & - & - & - & 0.0217 & - \\
45 & 0.17352 & 1.16011 & 0.19274 & - & - \\
60 & 0.17091 & 1.17882 & 0.1865 & - & - \\
75 & 0.17046 & 1.18021 & 0.17066 & - & - \\
90 & 0.16083 & 1.42537 & 0.1591 &
\end{tabular}

Table 3. Values of $\alpha_{x}, f_{x}, \alpha_{y}$ and $f_{y}$ of eqs. (8) and (9) at different impact angles.

\begin{tabular}{ccccc}
\hline Impact angle (degrees) & $\alpha_{x}$ & $f_{x}$ & $\alpha_{y}$ & $f_{y}$ \\
15 & 0.07803 & 2.02849 & - & 3.29346 \\
30 & 0.10083 & 2.00946 & 0.18763 & 3.87968 \\
45 & 0.16064 & 1.66344 & 0.18768 & 4.02227 \\
60 & 0.29973 & 1.5175 & 0.2184 & 4.25032 \\
75 & 0.41568 & 1.17189 & 0.23059 & 4.50602 \\
\hline
\end{tabular}




\section{References}

1. B. Robins, New Priniples of Gunnery (London, 1742).

2. L. Euler, Neue Grundsätze der Artillerie (1745); reprinted in Euler's Opera Omnia (Druck and Verlag Von B.G. Teubner, Berlin, 1922).

3. J.V. Poncelet, Coursde Mécanique Industrielle (Paris, 1829).

4. J.S. Uehara, M.A. Ambroso, R.P. Ojha, D.J. Durian, Phys. Rev. Lett. 90, 194301 (2003).

5. A.M. Walsh, K.E. Holloway, P. Habdas, J.R. de Bruyn, Phys. Rev. Lett. 91, 104301 (2003).

6. K.A. Newhall, D.J. Durian, Phys. Rev. E 68, 060301R (2003).

7. J.F. Boudet, Y. Amarouchene, H. Hellay, Phys. Rev. Lett. 96, 158001 (2006).

8. S.J. de Vet, J.R. de Bruyn, Phys. Rev. E 76, 041306 (2007).

9. M.A. Ambroso, C.R. Santore, A.R. Abate, D.J. Durian, Phys. Rev. E 71, 051305 (2005).

10. M. Hou, Z. Penga, R. Liua, Y. Wua, Y. Tiana, K. Lua, C.K. Chan, Sci. Technol. Adv. Mater. 6, 855 (2005).

11. X.-J. Zheng, Z.-T. Wang, Z.-G. Qiu, Eur. Phys. J. E 13, 321 (2004).

12. D.I. Goldman, P. Umbanhowar, Phys. Rev. E 77, 021308 (2008).

13. J.R. de Bruyn, A.M. Walsh, Can. J. Phys. 82, 439 (2004).

14. M.A. Ambroso, R.D.Kamien, D.J. Durian, Phys. Rev. E 72, 041305 (2005).

15. H. Katsuragi, D.J. Durian, Nat. Phys. 3, 420 (2007).

16. M.P. Ciamarra, A.H. Lara, A.T. Lee, D.I. Goldman, I. Vishik, H.L. Swinney, Phys. Rev. Lett. 92, 194301 (2004)

17. L.S. Tsimring, D. Volfson, in Powders and Grains, edited by R. Garcia-Rojo, H.J. Herrmann, S. McNamara, Vol. 2 (A.A. Balkema, Rotterdam, 2005) pp. 1215-1223.

18. A. Seguin, Y. Bertho, P. Gondet, J. Crassous, Europhys. Lett. 88, 44002 (2009).
19. F. Rioual, A. Valance, D. Bideau, Phys. Rev. E 62, 2450 (2000).

20. F. Rioual, A. Valance, D. Bideau, Europhys. Lett. 61, 194 (2003).

21. L. Oger, M. Ammi, A. Valance, D. Beladjine, Eur. Phys. J. E 17, 467 (2005).

22. J. Crassous, D. Beladjine, A. Valance, Phys. Rev. Lett. 99, 248001 (2007).

23. M. Ammi, L. Oger, D. Beladjine, A. Valance, Phys. Rev. E 79, 021305 (2009)

24. M. Nishida, M. Okumura, K. Tanaka, Granular Matter 12, 337 (2010).

25. S. Lee, D.B. Marghitu, Nonlinear Dyn. 57, 289 (2009).

26. R. Albert, M.A. Pfeifer, A.-L. Barabasi, P. Schiffer, Phys. Rev. Lett. 82, 205 (1999).

27. G. Hill, S. Yeung, S.A. Koehler, Europhys. Lett. 72, 137 (2005).

28. D. Kadau, H.J. Herrmann, Phys. Rev. E 83, 031301 (2011).

29. A. Seguin, Y. Bertho, P. Gondret, Phys. Rev. E 78, 010301 (2008).

30. P.A. Cundall, O.D.L. Strack, Geotechnique 29, 47 (1979).

31. X.-J. Zheng, N. Huang, Y.-H. Zhou, J. Geophys. Res. D 108, 4322 (2003).

32. J.F. Kok, N.O. Renno, Phys. Rev. Lett. 100, 014501 (2008).

33. D. Kadau, H.J. Herrmann, José S. Andrade Jr., Ascânio D. Araújo, Luiz J.C. Bezerra, Luis P. Maia, Granular Matter 11, 67 (2009)

34. D. Kadau, J.S. Andrade, J.H.J. Herrmann, Granular Matter 13, 219 (2011).

35. D. Kadau, J.S. Andrade, J.H.J. Herrmann, Eur. Phys. J. E 30, 275 (2009).

36. P. Umbanhowar, D.I. Goldman, Phys. Rev. E 82, 010301 (2010).

37. D. Lohse, R. Rauhé, R. Bergmann, D. van der Meer, Nature (London) 432, 689 (2004). 